\title{
Comparing Perception of Effort in Individual and Joint Action Contexts
}

\author{
Yahui Liang ${ }^{1}$, Thomas Wolf², Georgina Török ${ }^{2}$, Marcell Székely² \\ \& John Michael ${ }^{1,2 *}$
}

Cite as: Liang, Y., Wolf, T., Török, G., Székely, M., \& Michael, J. (2019, May 9). Comparing effort perception in individual and joint action contexts. Retrieved from osf.io/b7stn

DOI 10.17605/OSF.IO/B7STN

\footnotetext{
${ }^{1}$ Warwick University, Coventry, UK

${ }^{2}$ Central European University, Budapest, Hungary
}

*Corresponding Author: j.michael.2@warwick.ac.uk 


\begin{abstract}
In order to sustain cooperation, it is important that we have a sense that the distribution of efforts is fair. But how proficient are we at comparing our effort relative to that of others? Does the perception of our effort differ in individual and joint action contexts? To address these questions, we asked participants to squeeze a hand dynamometer at varying degrees of force to meet three target levels alone and with a partner. The results do not reveal a significant difference in the perception of effort between the two conditions. However, participants' estimation of their effort skewed towards half when they made partial contribution to the target and this effect was more pronounced in the joint action. Taken together, the findings suggest that participants might have applied heuristics when perceiving their effort and in addition, expected fairness in the effort contribution in a joint action context.

Keywords: effort, motivation, joint action, cooperation, fairness
\end{abstract}




\section{Đo My Part, You Do Yours: Comparing Perception of Effort in Individual and Joint Action Contexts}

\section{Introduction}

There are countless situations in everyday life in which we need to cooperate with a partner or a team to complete a task successfully or efficiently. From carrying a heavy table, to working in a group project and staffing the same departmental supervisor of an organisation, cooperation with others allow us to achieve goals that we might not be able to achieve alone (Tomasello, 2009; Tomasello, Melis, Tennie, Wyman, \& Herrmann, 2012). Yet, it is an open question how we compare our contributions within joint action contexts with the contributions of our partners/ other team members - even when the most pressing issue is to ensure that the joint goal is reached. For example, have you ever felt that you were bearing the bulk of the weight in carrying a heavy table and wished that your partner could exert more force? Most of us would also have had or heard grouses that a partner/ team member had not been "pulling his or her weight". These examples suggest that in order to sustain cooperation, it is important that we have a sense that the distribution of efforts is fair. Determining this sense requires us to compare our effort relative to others' but how proficient are we at making such comparisons?

Extensive research has been conducted in both economic and psychological game theory to understand and make predictions about interactions between individuals and their decisions, such as using the classic Prisoner's Dilemma game to investigate how individuals display cooperative and non-cooperative behaviour (e.g. Andreoni \& Miller, 1993; Colman, 2003; Rapoport, Chammah, \& Orwant, 1965; Sandoval, Brandstetter, Obaid, \& Bartneck, 2016). Rabin (2003) extended the standard game theory to incorporate social preferences, specifically fairness consideration, to model behaviour to describe why individuals tend to behave kindly to those they 
believed were kind to them. Stouten $(2005,2006)$ conducted experiments and found that participants had negative reactions towards others who did not make an equal and fair contribution to facilitate the provision of a public good, and argued that this was because individuals held the belief that everyone should contribute fairly. Studies have also shown that cooperative behaviour encourages more cooperation (Clark \& Sefton, 2001; Falk \& Fischbacher, 2006; Fehr \& Falk, 2002; Fischbacher, Gächter, \& Fehr, 2001).

Separately, there has been some research showing that the perception of a partner's effort promotes cooperative behaviour, e.g. by eliciting a sense of commitment (Michael, Sebanz, \& Knoblich, 2016a, 2016b) and enhances one's ability to resist temptation and persist longer in a joint action (Székely \& Michael, 2018). However, in determining whether the distribution of efforts is fair, there is little research on how proficient we are at comparing our effort relative to a partner's effort. Research has shown that we may wrongly attribute actions either to ourselves or others, depending on discrepancies between the predicted and actual sensory effects of our actions (Farrer \& Frith, 2002; Wegner, 2004). For example, Sato and Yasuda (2005) conducted an experiment in which participants pressed a button to generate a tone. They found that participants misjudged a tone as being generated by an external source if there was a delay between the expected and actual auditory feedback after they pressed a button. On the other hand, participants wrongly attributed a tone as generated by their action of pressing the button if the timing or the pitch of the tone was consistent with what they had predicted. In the same vein, Daprati et al. (1997) found that individuals mistook the experimenter's hand as their own when being shown a video of the experimenter's hand producing similar movements. Nonetheless in these experiments, participants performed the task alone (e.g. pressing the button) before assessing the "owner" of the 
action. Thus, there remains an open question on how we assess our effort relative to others' in a joint action context.

The present study investigated how well we assess our own effort level, and whether we exhibit any biases in this regard when working with a partner compared to when working alone. Latané, Williams, and Harkins (1979) asked participants to make noises individually and in actual/pseudo-groups and found that individuals judged the noises they made to be louder than those of others. In line with this effect, our effort may seem greater to us than it actually is simply because it is more salient. Therefore, one hypothesis is that participants will assess their effort level to be greater than it actually is when they are working with a partner. On the other hand, Diehl and Stroebe $(1987,1991)$ conducted individual and group brainstorming sessions, and consistently found that individuals perceived working in a group to be more enjoyable than working alone. As such, an alternative hypothesis is that participants will assess their effort to be lower than actual when working with a partner, as they may find the joint task more motivating and less effortful.

In order to test the two hypotheses, we devised a paradigm in which the concept is analogous to the real-life example of carrying a table - an individual first carried a table alone and feels the full weight of the table, say $100 \mathrm{~N}$. The same individual then carried a load again under two scenarios i.e. a lighter table weighing $50 \mathrm{~N}$ alone or the same $100 \mathrm{~N}$-table together with a partner exerting $50 \mathrm{~N}$ each. Even though the actual force exerted by the individual in the second round is exactly half compared to that in the first round under both scenarios, the individual's perception of own effort might be more or less than half and different between the two scenarios.

To implement the paradigm, we asked participants to squeeze a hand dynamometer at varying degrees of force to meet various targets individually and jointly with a partner. We employed grip force as a measurement of effort, as it has been similarly used in several past 
experiments to study effort and its relationship with reward, motivation, emotion and delay (e.g. Bonnelle, Manohar, Behrens, \& Husain, 2015; Chong et al., 2017; Cléry-Melin et al., 2011; Kurniawan et al., 2010; Prévost, Pessiglione, Météreau, Cléry-Melin, \& Dreher, 2010). Following the example of carrying a table, participants first squeezed the dynamometer to hit a target level. They then repeated the task either alone but with the computer adding a certain amount to their force as they reached the target (Computer condition), or reached the target jointly with a partner (Partner condition). Participants were then asked to estimate their effort exerted in the second round compared to the first round. Based on the hypothesis that we overestimate our own effort contribution in joint action contexts, we predicted that participants will overestimate their effort in the second round to a greater extent in the Partner condition than in the Computer condition. If, on the other hand, we underestimate our effort in joint action contexts, then participants should underestimate their effort in the second round to a greater extent in the Partner condition than in the Computer condition.

\section{Methods}

The study was pre-registered on AsPredicted.org, with the sample size, planned analyses and data exclusion criteria specified. The pre-registration document can be accessed at https://aspredicted.org/blind.php?x=kh3wa9.

\subsection{Participants}

Using G*Power 3.1 (Faul, Erdfelder, Buchner, \& Lang, 2009), it was determined that a sample size of 34 would provide $80 \%$ statistical power for detecting a medium effect size (Cohen's $d=0.50$ ), assuming a two-tailed t-test and an alpha level of 0.05 . In anticipation of possible exclusions, a total of 38 participants (18 females, 20 males) were recruited via the University of Warwick online sign-up system for research. All participants signed informed consent prior to the 
experiment and received up to $£ 10$ for their participation. They were also informed that they could choose to leave the experiment at any time. The experiment was approved by the University Department Ethics Committee.

Of the 38 participants, two were excluded due to a problem with the task program in the midst of the experiment, and another two were excluded due to failure to perform in the rating task (explained further in Section 2.5). Therefore, only 34 participants were included in the reported analyses (17 females, 17 males; age range: $19-49$, mean age $=26$ years, $S D=6$ years $)$.

\subsection{Design}

A 2 x 3 within-subject design was employed, comprising two conditions i.e. Computer and Partner and three target levels at 25\%,40\% and 55\%. Pilot tests were conducted to ensure that the target levels were effortful yet manageable by the participants throughout the experiment. Each experiment involved a pair of participants. To avoid any confound caused by the presence of an opposite gender on the behaviour and performance of the participants, participants of the same gender were paired in the experiment.

\subsection{Apparatus and materials}

Participants were each seated in front of a laptop with a pair of ear defenders (34dB SNR, MPow). Each pair of participants was positioned across a table from each other with an occluder between them (see Figure 1A). Participants' grip forces were recorded using a pair of hand dynamometers (HD-BTA, Vernier Software \& Technology, USA) with a sample rate of 25Hz. The recorded signal was digitalised and fed in real-time into the experimenter's laptop running the task program. To minimise discomfort from repeated grips, foam padding was added to cushion the side of the dynamometer that would be resting against the participant's palm during the grip task 
(See Figure 1B). The presentation of the visual information and data recording were implemented using a custom Max MSP patch.

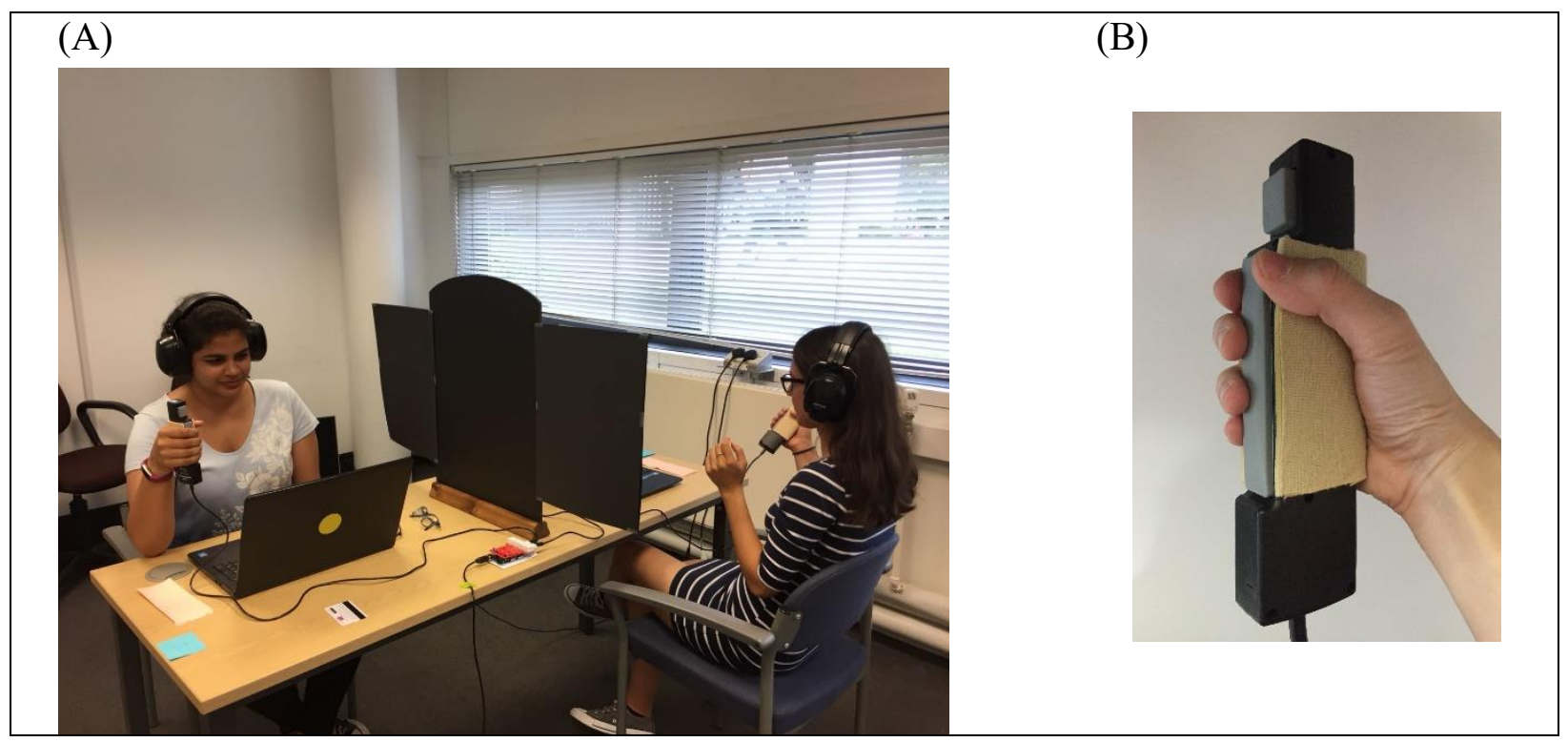

Figure 1. (A) Experimental setup with a pair of participants. (B) Dynamometer with form padding on the side resting against the palm when gripping.

\subsection{Procedure}

Participants were first informed that the objective of the experiment was to measure how well they perceived their effort and they would be required to squeeze the hand dynamometer to varying degrees of force to meet a range of targets. They were also given a brief demonstration on how to grip the hand dynamometer and to keep the device upright when squeezing. Participants were told to use their dominant hand throughout the experiment.

\subsubsection{Force calibration}

Each participant's maximum voluntary contraction (MVC) was established so that the target levels could be normalised across individuals as well as between the two participants within each pair. Participants were given instructions on their screen to squeeze the dynamometer as hard as they could and were told to release their grip only when the screen message changed to "Stop" 
3 s later. The calibration procedure was repeated once. Participants' individual MVC was calculated by taking the average of their force values recorded over the two 3 -s periods. This calibration approach was used as participants had to keep their grip force steady for a 3-s period in the experimental task.

The target levels were set at $25 \%, 40 \%$ and $55 \%$ of the higher MVC between the two participants within each pair. In addition, a multiplier factor (ratio of the higher to lower MVC) was applied to the force recorded from the participant with the lower MVC. This was implemented to ensure that the same target level would feel similarly effortful to both participants relative to their own MVC. Table 1 shows an example of how the 40\%-target was set for a pair of participants with MVCs of $200 \mathrm{~N}$ and $100 \mathrm{~N}$ respectively. Participants were informed of this normalisation procedure within each pair, in order to minimise any bias that might have arisen in their judgements from having perceived their partner to be physically more or less strong.

Table 1

Table Showing Example of How the 40\% Target Was Set for a Pair of Participants Based On Their MVCs.

\begin{tabular}{lllll}
\hline Participant & Individual MVC & $\begin{array}{l}\text { Target } \\
\text { (at 40\%-level) }\end{array}$ & $\begin{array}{l}\text { Actual } \\
\text { exerted }\end{array}$ & $\begin{array}{l}\text { force } \\
\text { force recorded and used } \\
\text { for analysis }\end{array}$ \\
\hline $\mathrm{A}$ & $\begin{array}{l}200 \mathrm{~N} \\
\text { (higher } M V C)\end{array}$ & $80 \mathrm{~N}$ & $80 \mathrm{~N}$ & $80 \mathrm{~N}$ \\
$\mathrm{~B}$ & $\begin{array}{l}100 \mathrm{~N} \\
\text { (lower } M V C)\end{array}$ & $80 \mathrm{~N}$ & $40 \mathrm{~N}$ & $\begin{array}{l}80 \mathrm{~N} \\
\left(40 \mathrm{~N} \times 2^{\wedge}\right)\end{array}$ \\
\hline
\end{tabular}

Note. ${ }^{\wedge}$ Participant B's actual force is multiplied by a factor of $2(200 \mathrm{~N} \div 100 \mathrm{~N})$.

\subsubsection{Training phase}

Following force calibration, participants underwent nine randomised training trials (three trials per target level), so that they were familiar with the visual stimuli and had experience of their physical force exerted at each target level. 
At the start of the training phase, participants were given a brief written guide to read, including sample screenshots, on what to do during the training trial (see Appendix A). In each training trial, participants first saw an instruction on the screen to get ready. An instruction to "Increase force until target is reached" was then displayed for 3 s. Upon seeing this message, participants were required to squeeze the dynamometer as soon as possible, until their force level, shown in the form of a mint green coloured column via a real-time feedback on the screen, reached within the green target box (see Figure 2A). Next, participants were instructed to maintain their force at the target level for another $3 \mathrm{~s}$.

In the study by Kurniawan et al. (2010) using hand dynamometer, participants were required to reach the target within $2 \mathrm{~s}$ after the stimulus onset. Prévost et al. (2010) also found that said that participants took less than $2 \mathrm{~s}$ to reach the various target levels. However, participants could exceed the required target level by any amount in these experiments. In contrast, the current experiment required participants to keep their force level within the green target box that was set at $100 \%$ to $120 \%$ of the target level. As such, they were given a longer 3 -s period to reach the target. Allowing the buffer for the participants' force to fluctuate between $100 \%$ to $120 \%$ of the target level was so that they could devote attention to assessing the physical effort they were exerting. 


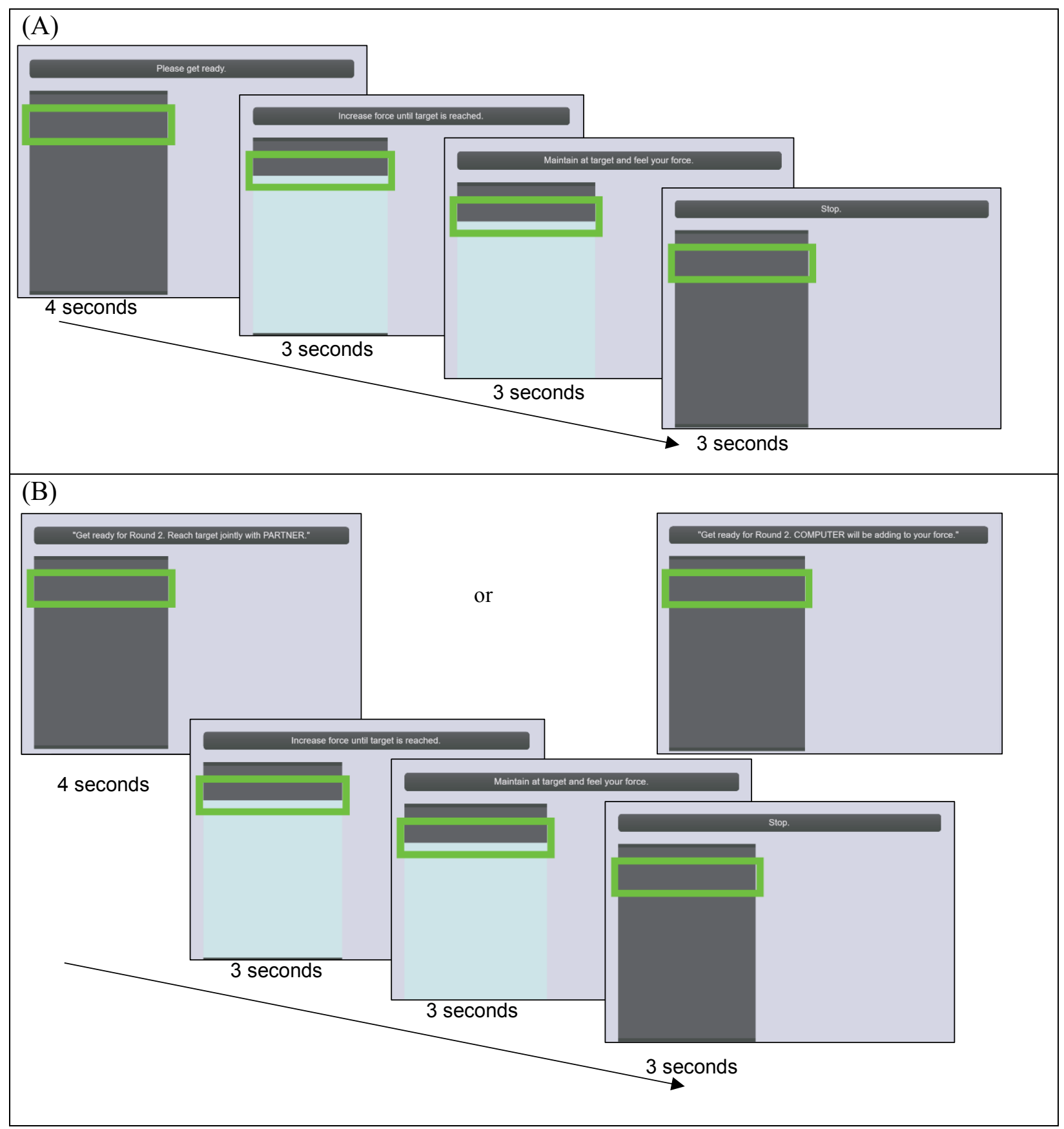

Figure 2. (A) Trial structure in learning phase and Round 1 of experimental phase. Participants squeezed the dynamometer and saw visual feedback of their force level (coloured column in mint green). They were required to increase their force reach the target box within $3 \mathrm{~s}$ and maintain their force at target for another $3 \mathrm{~s}$. (B) Trial structure of Round 2 in experimental phase. Participants were informed whether they were in the Partner condition or Computer condition. 


\subsubsection{Experimental phase}

After the training phase, participants were similarly given a guide what to do during the main experimental trial, including sample screenshots, to read through (see Appendix B). To ensure understanding of the experiment requirements, the instructions were also explained verbally and participants were prompted to ask any questions before they began. The briefing on the main experiment was given after the training trials, so that participants can rest their dominant hand before the main experiment.

The main experiment had a total of 48 trials under two conditions i.e. Partner and Computer. In each condition, there were two blocks of 12 trials each (four trials per target level). Each trial comprised two rounds of grip task and a rating task.

In Round 1 of the grip task, participants had $3 \mathrm{~s}$ to squeeze the dynamometer until their force reached the target and were required to maintain their force at target for another $3 \mathrm{~s}$. Round 1 was similar to the training trial. Participants were required to mentally note the physical force exerted in Round 1. Participants then repeated the task in Round 2 depending on the condition (see Figure 2B). In the Partner condition, participants were required to reach the target jointly with their partner seated across the table. In the Computer condition, participants were told that the computer would be adding some amount of force as they reached the target. To avoid any confounding effect from participants having the impression that they were working with a virtual partner, it was emphasised to the participants that they would be still be performing the grip task alone in Round 2 in the Computer condition. However, unbeknownst to participant, the force added by the computer in Round 2 was actually from their partner. This manipulation ensured that the participants would experience similar complementary forces in both conditions, and that the only 
difference between the two conditions was the belief as to whether their partner was also exerting an effort together with them.

At the end of Round 2, participants were asked to rate their force exerted in Round 2 as a percentage of their force exerted in Round 1, by sliding a vertical scale ranging from $0 \%$ to $120 \%$ on the screen with their laptop mousepad (see Figure 3). Participants' ratings were also shown numerically so that they could check their response before clicking a round button to confirm. During the experiment, participants wore ear defenders and had the occluder between them to prevent visual contact or influence from any noise made by the partner. They were told that their incentives were based on how well they met the targets in both rounds of the grip task and how accurate they were in the rating task, but were not informed about the precise performance and reward computation.

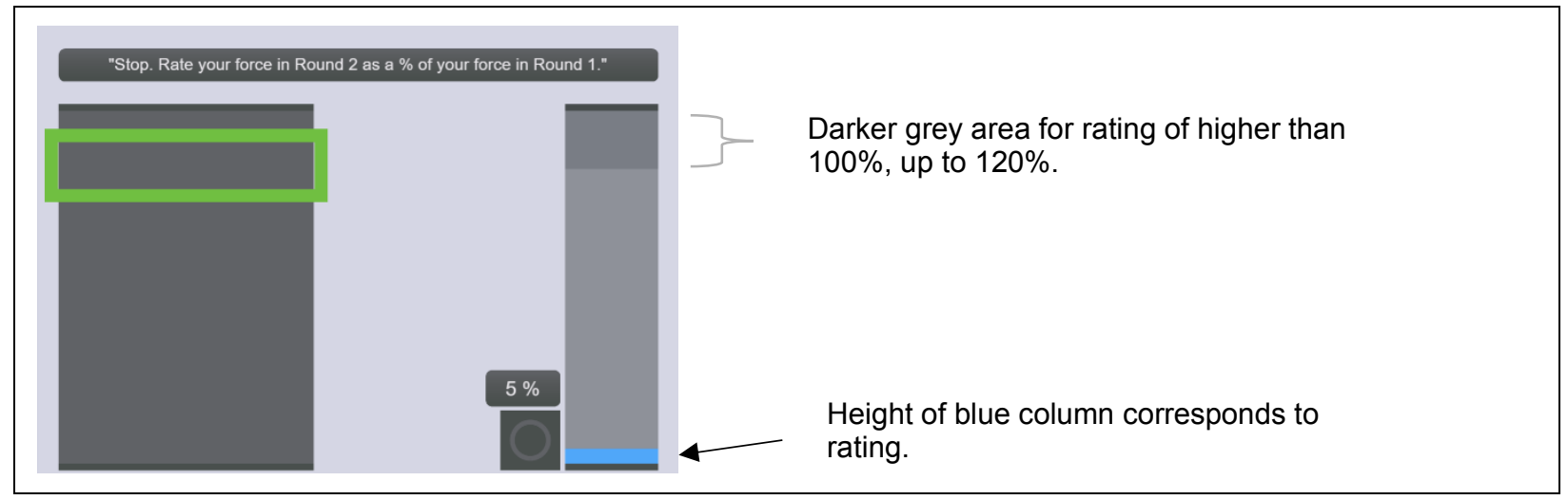

Figure 3. Rating task of the experimental trial. Participants could slide a vertical scale from $0 \%$ to $120 \%$ to indicate their estimated Round 2 effort as a percentage of Round 1 effort. Example in the figure shows a rating at $5 \%$.

After one block of 12 consecutive trials, participants were given a break of $30 \mathrm{~s}$ before being prompted to click a button when they felt ready to continue. To minimise fatigue, they were 
told that they could take a break for as long as they needed before proceeding to the next block. The next block started after both participants were ready.

The order of the Partner and Computer conditions was counterbalanced to avoid any sequence effects. Within each block, the target level was random across trials. However, participants either completed two consecutive blocks in the Computer condition first before two consecutive blocks in the Partner condition, or vice versa. This was intended to prevent the participants from getting confused and failing to differentiate between the conditions.

\subsection{Data analysis}

The dependent variable considered was the judgement error between the participants' subjective rating (i.e. their Round 2 effort as a percentage of their Round 1 effort) and the actual rating. The actual rating was derived by taking the average of the force exerted by the participants during the second 3-s period during Round 2 divided by the corresponding average force in Round 1 (i.e. when they had to maintain their force at target). The judgement error in each trial, expressed as a ratio, was determined as follows:

Judgement error $=\frac{\text { Subjective rating }(\%)-\text { Actual rating }(\%)}{\text { Actual rating }(\%)}$,

where Actual rating $=\frac{\text { Average force exerted during the } 3-\mathrm{s} \text { "maintain" period in Round } 2}{\text { Average force exerted during the } 3 \text {-s "maintain" period in Round } 1}$

Based on the formula above, if a participant's subjective rating is $50 \%$ and actual rating is $40 \%$, the judgement error will be 0.25 .

The exclusion rules specified in the pre-registration were to (i) exclude trial for both participants of a pair, if their combined forces in Round 2 were lower than half of the trial's target; and (ii) exclude participants if they do not meet their Round 1 targets for more than $50 \%$ of the trials. 
The share of effort put in by each participant in Round 2, based on the actual force (in N) exerted by the participant as a ratio of the combined force (in N) exerted by the participant and partner in Round 2, was also derived for an exploratory analysis on the correlation between judgement error and share of effort.

As mentioned in Section 2.1, two participants in the same session did not complete the experiment due to a problem with the task. Another two participants did not perform properly in the rating task either because they misunderstood the instruction or were unwilling. One of them provided similar ratings around $100 \%$ regardless of the effort in Round 2, while the other had judgement errors that were abnormally large and many times more than those of the other participants. For example, this participant gave a rating of $20 \%$ in one trial, when the actual percentage of her Round 2 over Round 1 effort was only about $0.0017 \%$ i.e. resulting in a judgement error of about 115 . There were also a small portion of trials $(0.73 \%$ of data) where the participants failed to meet at least half the trial's target in Round 1 or did not provide a rating at the end of Round 2 because they accidentally missed the signal. Assessment was done to exclude the abovementioned participants/trials, in addition to the exclusions specified in the preregistration, prior to performing any analyses. Separately, the data of one trial (affecting both participants in the same session) was missing due to failure of the apparatus/task program to record the data.

\section{Results}

Do judgement errors differ between the Computer and Partner conditions? Results show that they do not. On average, participants overestimated i.e. perceived their Round 2 effort to be higher than actual in both conditions (see Figure 4). Although the mean judgement error was higher in the Partner condition $(M=0.16, S D=0.47)$ than in the Computer condition $(M=0.12, S D=$ 
0.36 ), a paired-samples $t$-test revealed no significant difference between the conditions, $t(33)=$ $0.65, p=.520$, Cohen's $d=0.111$.

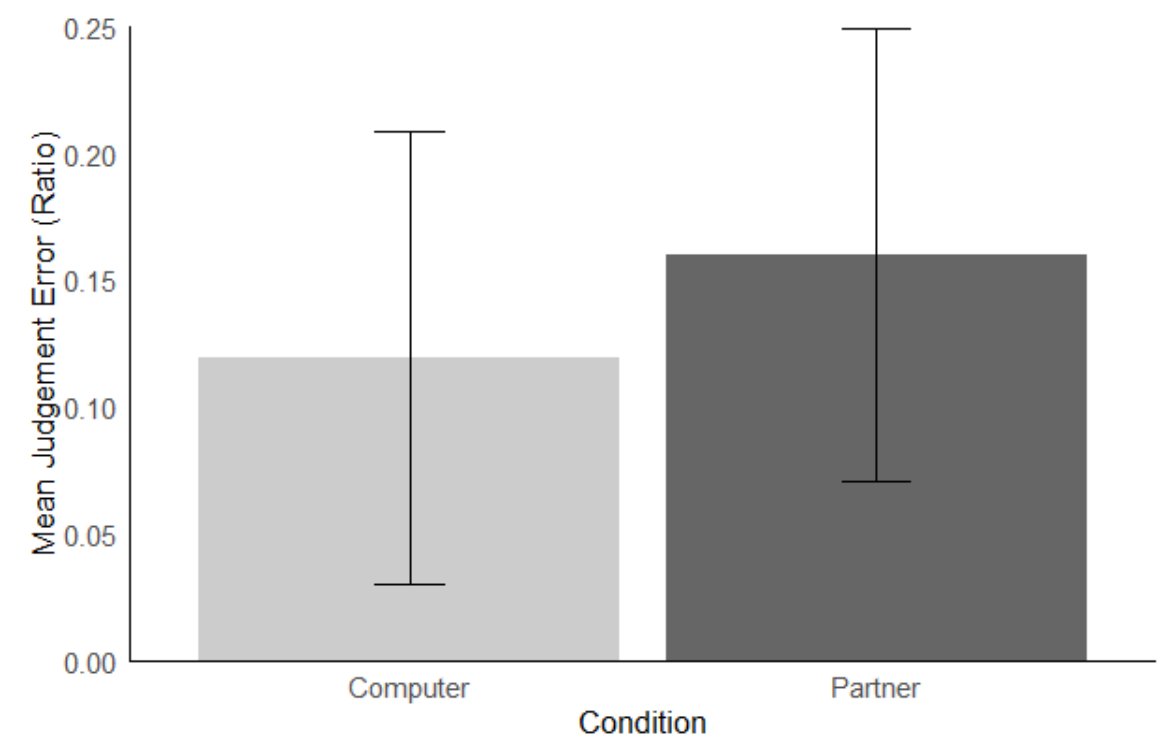

Figure 4. Mean judgement error (expressed as a ratio) in the Computer and Partner conditions. Error bars represent the within-subject confidence intervals (following the method proposed by Morey, 2008; cf. Cousineau, 2005).

A 2 (condition) x 3 (target level) repeated measures ANOVA confirmed that the main effect of condition upon judgement error was non-significant, $F(1,33)=0.413, p=.525$. However, the main effect of target level was significant, $F(2,66)=22.49, p<.0001$, indicating that the judgement errors generally increased with the target level.

Figure 5 plots the mean judgement errors at each target level in both conditions. In the Computer condition, participants underestimated their Round 2 effort on average. The mean judgement errors changed from under-estimation at the $25 \%$-target level to over-estimation at the 40\%- and 55\%- target levels. In the Partner condition, participants overestimated their Round 2 effort on average across all three target levels, and the increase in the judgement errors were relatively similar between any two target levels. Post-hoc paired-samples $t$-tests with Bonferroni 
correction showed that in the Computer condition, mean judgement errors at the $25 \%$-target level $(M=-0.055, S D=0.37)$ were significantly different from those at the $40 \%$-target level $(M=0.16$, $S D=0.44), t(66)=4.70, p=.0006$ and 55\%-target level $(M=0.25, S D=0.37), t(66)=6.55, p$ $<.0001$, but were not significantly different between the $40 \%$ - and $55 \%$ - target levels, $t(66)=1.86$, $p=.184$. In the Partner condition, mean judgement errors at the $25 \%$-target level $(M=0.040, S D$ $=0.49)$ were not significantly different from those at the $40 \%$-target level $(M=0.17, S D=0.50)$, $t(66)=2.18, p=.188$ but were significantly different from those at the $55 \%$-target level $(M=0.27$, $S D=0.55), t(66)=3.71, p=.001$. There was no significant difference between the mean judgement errors at the $40 \%$ - and 55\%-target levels, $t(66)=1.53, p=.321$. In addition, there was no significant interaction between condition and target level, $F(2,66)=0.894, p=.414$.

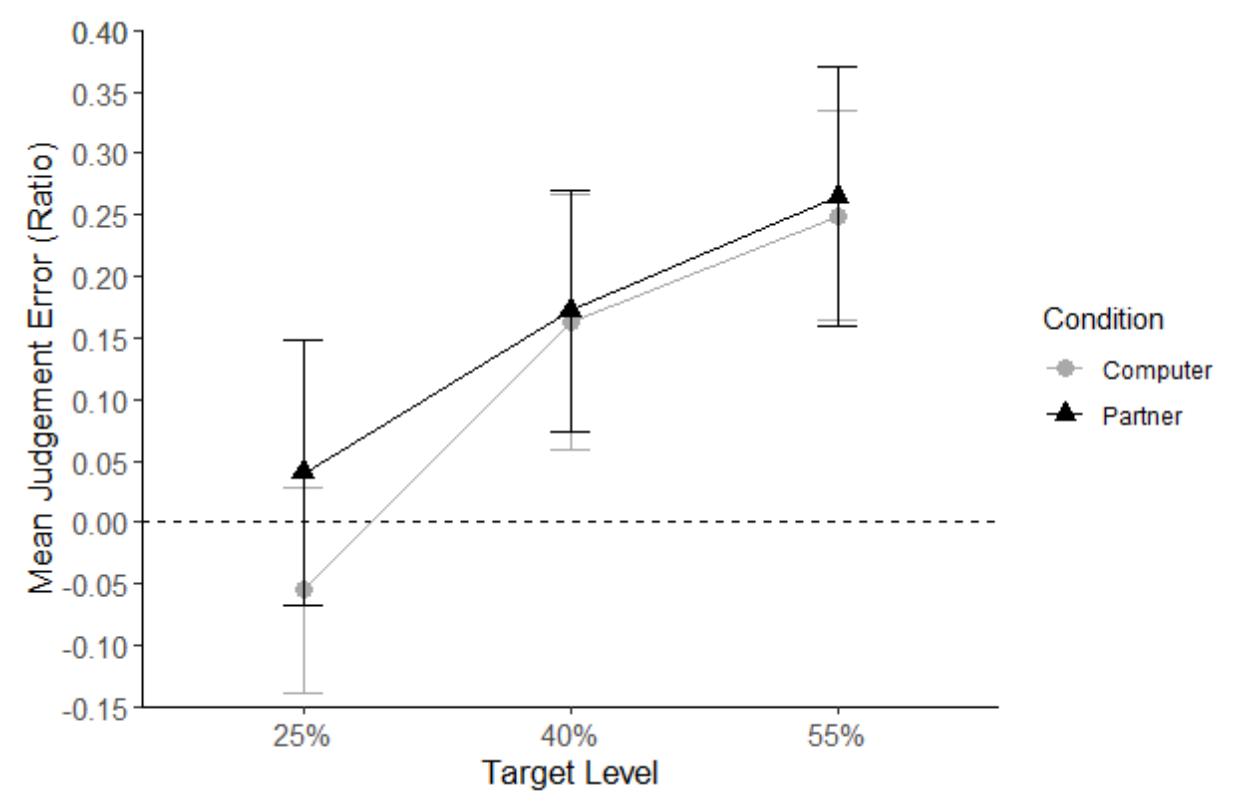

Figure 5. Mean judgement error (expressed as a ratio) at each target level in the Computer and Partner conditions. Error bars represent the within-subject confidence intervals according to Morey (2008). Dotted line represents zero judgement error and areas above and below this line reflect over- and under-estimation of Round 2 over Round 1 effort. 
While the results did not reveal a significant effect of condition, Figure 5 suggests that participants' judgement errors were relatively greater in magnitude in the Computer condition at the lowest target level compared to the Partner condition, while the judgement errors were higher in the Partner condition than the Computer condition at the higher target levels by a smaller extent. To investigate whether participants made greater absolute judgement errors in one condition over the other, a paired-samples $t$-test was run on the magnitude of the errors i.e. regardless of whether the errors were over- or under-estimation. Results show that the absolute judgement errors made in the Computer condition $(M=0.40, S D=0.22)$ did not differ significantly from those made in the Partner condition $(M=0.47, S D=0.31), t(33)=1.20, p=.238, d=0.206$.

As same-gender pairing was employed in the experiment, a three-way ANOVA was conducted to check for any effect of gender. The results did not reveal any significant effect of gender upon judgement error, $F(1,32)=0.213, p=.648$. There was also no significant interaction between gender and condition, $F(1,32)=0.151, p=.700$, between gender and target level $F(2$, $64)=0.526, p=.594$ and among the three factors, $F(2,64)=1.329, p=.272$.

An exploratory analysis was conducted to investigate whether judgement errors were correlated with the share of effort towards the target in Round 2. Figure 6 plots the average judgement error as a function of the individual's share of effort. In both conditions, the mean judgement errors were observed to be negatively-correlated with the share of effort. This indicates that participants tended to overestimate when their share of effort was less than half, and the judgement error increased as their share decreased towards zero percent. Perception of effort was the most accurate when the share of effort between the two participants were about equal. Participants tended to underestimate if their share of effort was more than half and the judgement error increased as the share increased. This relationship appeared stronger in the Partner condition. 
Participants' individual correlation coefficients were derived from their trial-level data. Spearman's correlation coefficient $r$ was computed because for some participants' trial-level data deviated from normality based on the Shapiro-Wilk test. The results confirmed a higher correlation $($ mean $r(33)=-.342, S D=0.348)$ in the Partner condition compared the Computer condition (mean $r(33)=-.218, S D=0.387)$. A paired-samples $t$-test showed a significant difference, $t(33)=2.45$, $p=.020, d=0.407$.

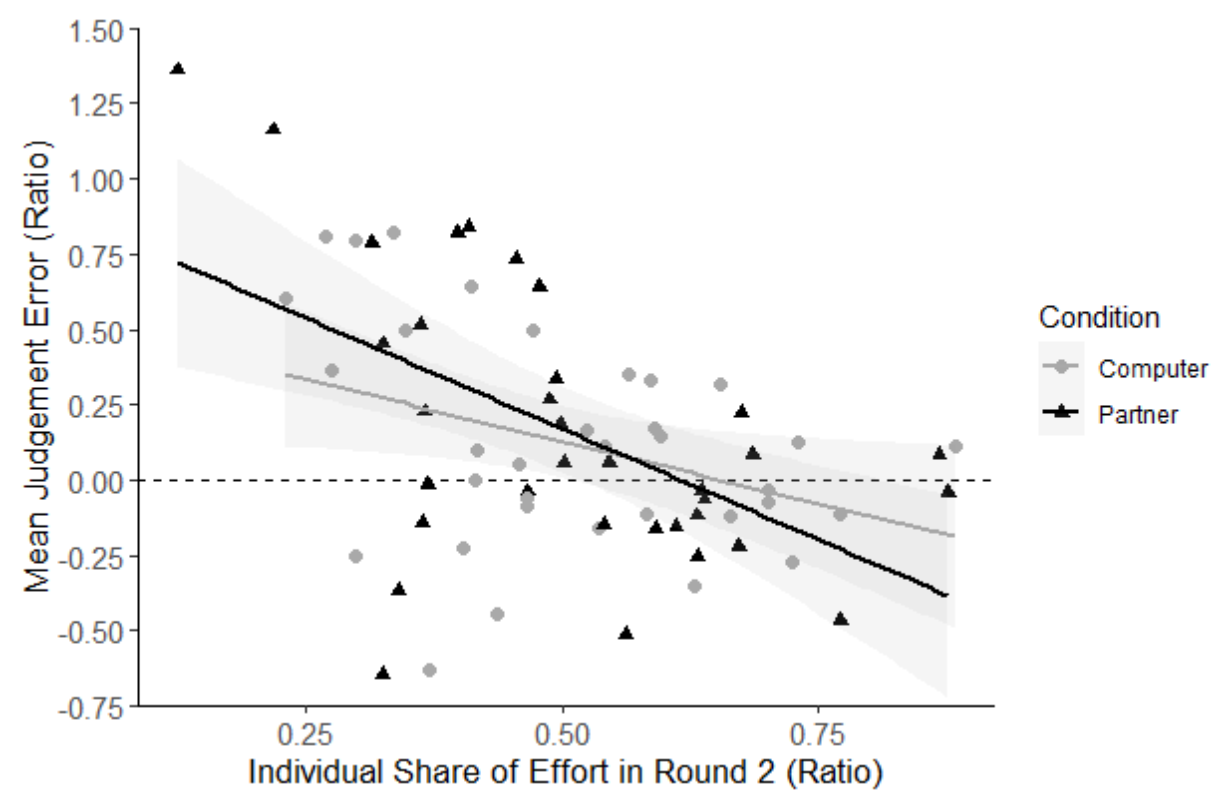

Figure 6. Correlation between mean judgement error (expressed as a ratio) and individual's share of effort in Round 2 (expressed as a ratio). Shaded areas show the respective 95\% CI. Dotted line represents zero judgement error and areas above and below this line reflect over- and underestimation of Round 2 effort as a percentage of Round 1 effort.

From the plot in Figure 6, it is apparent that two participants in the Partner condition had particularly high mean judgment errors (as indicated by the two triangles in the upper left of the plot). In order to probe whether the difference between the two conditions may have been driven by these two data points, we also ran an exploratory analysis on the individual correlation 
coefficients excluding datapoints from the two participants. The results revealed that the correlation between the mean judgement error and share of effort remains significantly higher in the Partner condition with (mean $r(31)=-0.317, S D=0.342)$ than in the Computer condition (mean $r(31)=-0.201, S D=0.380)$. A paired-samples t-test similarly showed a significant difference between the two conditions, $t(31)=2.30, p=.028$.

\section{Discussion}

The present study investigated whether participants exhibited any biases in assessing their effort when working with a partner compared to when working alone. In terms of numerical difference, participants were observed to overestimate their effort across all target levels in the Partner condition, but only overestimated in the tasks that were highly effortful in the Computer condition. When they overestimated, they also overestimated to a greater extent in the Partner condition. The difference in judgement errors between the two conditions, however, was not significant, and therefore does not provide evidence in support of either of the two competing hypotheses that were pitted against each other in this experiment. Thus, it remains inconclusive whether we exhibit any biases when assessing our effort level, and what effect joint action contexts may have upon such assessments.

At the end of the experiment when participants were debriefed, many were surprised that the force that was supposedly added by the computer was actually from their partner. One participant even remarked that "the computer did most of the work". Nonetheless, a small handful of participants shared that they had suspected the reverse i.e. that the partner's force was from the computer algorithm. Only one participant suspected that the computer's force was from the partner. However, he had thought that the computer's behaviour was programmed based on the partner's force exerted in the preceding trial, when in fact the force added by the "computer" was the 
partner's real-time force in the joint task. Although the experimental manipulation was successful for most of the participants, the suspicion by the few participants on the set-up of the two conditions might have affected the findings especially given that the sample size was not large. As the feedback from the participants during the debrief was not systematically solicited and recorded, a further analysis using only the responses from the subset of participants who were totally naïve to the experimental manipulation could not be conducted. Hence, the experimental results should be validated further.

Participants made a smaller overestimation of their effort at the lowest target level compared to the highest target level in the Partner condition. In the Computer condition, they underestimated their effort at the lowest target level instead while making about the same amount of overestimation at the highest target level compared to the Partner condition. Taken together, the results suggest that participants estimated their effort to be relatively smaller when the target was easy. One possible explanation for this is that the participants misattributed their self-generated force to the computer or partner because the visual feedback of their force level vis-à-vis the target did not match their predictions (Farrer \& Frith, 2002; Sato \& Yasuda, 2005; Wegner, 2004). The target at $25 \%$ was easily achieved. When the pair of participants jointly reached this target in Round 2 of the trial, their combined force level tended to rise much faster and often overshot the target box at the start. Hence, if participants saw on the visual display that the combined force level in Round 2 at the $25 \%$ target level had increased at a much faster rate than what they had predicted or expected (because each only exerted a small amount of force), they might have been more inclined to think that the result was due to the external source and not their own force.

The exploratory analysis showed that participants' estimation of their effort was most accurate when their share of effort with the partner/computer in Round 2 was about half. Their 
judgement tends towards underestimation when their share of effort was more than half and overestimation when their share of effort was less than half, and this effect is more pronounced in the Partner condition. The findings suggest that participants' responses were skewed towards 50\%. One possible interpretation is that participants used heuristics and anchored the estimation of their Round 2 over Round 1 effort conveniently around 50\% because exerting about half their Round 1 effort should get them to the target in Round 2. Anchoring is a bias where one's judgement is systematically biased toward a starting point arising from a value that is initially presented (Tversky \& Kahneman, 1974). As a result, when participants put in more than half towards the target in Round 2, they might have estimated their response by incrementing from the 50\% starting point instead of comparing with their $100 \%$-effort in Round 1, leading to an underestimation of their actual effort. Conversely, when their share of effort was less than half, they might have adjusted their response from the 50\% starting point resulting in overestimation of their effort. However, anchoring cannot fully explain why the bias is more pronounced in the Partner condition. One possible speculation is that when working with a partner, participants expect fairness (Rabin, 2003; Stouten, 2005, 2006) in the contribution of effort towards the joint target, and this expectation accentuated the bias towards the $50 \%$ norm.

The present study implemented a paradigm that facilitates the investigation into how well we compare our effort relative to others' and suggests that the difficulty of a task and expectation of a partner's contribution may influence our judgement. This study has theoretical and practical implications, providing insight into how our perception of our own and our partners' efforts may be biased in joint action contexts. Any findings in this direction may be applied in real-life contexts to contribute to sustaining cooperation, and possibly offer solutions to reducing undue resentment from perceived unfairness in effort contributions. 
However, the present study had important limitations. One limitation was that the variability in the reaction times and the shares of contribution by the partner both across the trials within each experiment session and across the experimental sessions. If the reaction times between a pair of participants were relatively different, there is a tendency that the faster participant ends up putting more force towards the joint target in Round 2 and leaving less room for the slower partner to contribute. Even if the participants within a pair had similar reaction times, their relative share of contribution at the same target levels were also varied in different trials. To overcome the limitation, the experimental design could be tweaked to hold the partner's contribution constant, either by replacing the partner with a computer algorithm or by using a confederate to act as the partner for all the experimental sessions. This would ensure that the share of effort contributed by the participants in different experimental sessions could be kept constant at one or a few fixed levels, to better measure the estimation of their effort. Another improvement to the current design would be to frame the question differently. Comparing one's Round 2 effort as a percentage of Round 1 effort places an extra onus on the participants to remember the force they had exerted in Round 1. Alternatively, participants could be asked to estimate their partner's contribution directly in Round 2. This question would also provide a better basis for drawing inferences into how well we perceive a partner's effort.

Future research should also look into how a pre-committed distribution of effort e.g. deciding one party should contribute $60 \%$ while the other does $40 \%$ would affect how we compare relative efforts. A further interesting question for future research is whether and how other cues of a partner's effort e.g. looking at the partner's arm or facial expression influence our assessment of the partner's effort. 


\section{References}

Andreoni, J., \& Miller, J. H. (1993). Rational cooperation in the finitely repeated prisoner's dilemma: Experimental evidence. The Economic Journal, 103(418), 570-585.

Bonnelle, V., Manohar, S., Behrens, T., \& Husain, M. (2015). Individual differences in premotor brain systems underlie behavioral apathy. Cerebral Cortex, 26(2), 807-819.

Chong, T. T.-J., Apps, M., Giehl, K., Sillence, A., Grima, L. L., \& Husain, M. (2017). Neurocomputational mechanisms underlying subjective valuation of effort costs. PLoS Biology, 15(2), e1002598.

Clark, K., \& Sefton, M. (2001). The sequential prisoner's dilemma: evidence on reciprocation. The Economic Journal, 111(468), 51-68.

Cléry-Melin, M.-L., Schmidt, L., Lafargue, G., Baup, N., Fossati, P., \& Pessiglione, M. (2011). Why don't you try harder? An investigation of effort production in major depression. PloS One, 6(8), e23178.

Colman, A. M. (2003). Cooperation, psychological game theory, and limitations of rationality in social interaction. Behavioral and Brain Sciences, 26(2), 139-153.

Cousineau, D. (2005). Confidence intervals in within-subject designs: A simpler solution to Loftus and Masson's method. Tutorials in Quantitative Methods for Psychology, 1(1), 42-45.

Daprati, E., Franck, N., Georgieff, N., Proust, J., Pacherie, E., Dalery, J., \& Jeannerod, M. (1997). Looking for the agent: an investigation into consciousness of action and self-consciousness in schizophrenic patients. Cognition, 65(1), 71-86.

Diehl, M., \& Stroebe, W. (1987). Productivity loss in brainstorming groups: Toward the solution of a riddle. Journal of Personality and Social Psychology, 53(3), 497. 
Diehl, M., \& Stroebe, W. (1991). Productivity loss in idea-generating groups: Tracking down the blocking effect. Journal of Personality and Social Psychology, 61(3), 392.

Falk, A., \& Fischbacher, U. (2006). A theory of reciprocity. Games and Economic Behavior, 54(2), 293-315.

Farrer, C., \& Frith, C. D. (2002). Experiencing oneself vs another person as being the cause of an action: the neural correlates of the experience of agency. Neuroimage, 15(3), 596-603.

Faul, F., Erdfelder, E., Buchner, A., \& Lang, A.-G. (2009). Statistical power analyses using G* Power 3.1: Tests for correlation and regression analyses. Behavior Research Methods, 41(4), 1149-1160.

Fehr, E., \& Falk, A. (2002). Psychological foundations of incentives. European Economic Review, 46(4-5), 687-724.

Fischbacher, U., Gächter, S., \& Fehr, E. (2001). Are people conditionally cooperative? Evidence from a public goods experiment. Economics Letters, 71(3), 397-404.

Kurniawan, I. T., Seymour, B., Talmi, D., Yoshida, W., Chater, N., \& Dolan, R. J. (2010). Choosing to make an effort: The role of striatum in signaling physical effort of a chosen action. Journal of Neurophysiology, 104(1), 313-321.

Latané, B., Williams, K., \& Harkins, S. (1979). Many hands make light the work: The causes and consequences of social loafing. Journal of Personality and Social Psychology, 37(6), 822.

Michael, J., Sebanz, N., \& Knoblich, G. (2016a). Observing joint action: Coordination creates commitment. Cognition, 157, 106-113.

Michael, J., Sebanz, N., \& Knoblich, G. (2016b). The sense of commitment: A minimal approach. Frontiers in Psychology, 6, 1968. 
Morey, R. D. (2008). Confidence intervals from normalized data: A correction to Cousineau (2005). Reason, 4(2), 61-64.

Prévost, C., Pessiglione, M., Météreau, E., Cléry-Melin, M.-L., \& Dreher, J.-C. (2010). Separate valuation subsystems for delay and effort decision costs. Journal of Neuroscience, 30(42), 14080-14090.

Rabin, M. (1993). Incorporating fairness into game theory and economics. The American economic review, 1281-1302.

Rapoport, A., Chammah, A. M., \& Orwant, C. J. (1965). Prisoner's dilemma: A study in conflict and cooperation (Vol. 165): University of Michigan press.

Sandoval, E. B., Brandstetter, J., Obaid, M., \& Bartneck, C. (2016). Reciprocity in human-robot interaction: a quantitative approach through the prisoner's dilemma and the ultimatum game. International Journal of Social Robotics, 8(2), 303-317.

Sato, A., \& Yasuda, A. (2005). Illusion of sense of self-agency: discrepancy between the predicted and actual sensory consequences of actions modulates the sense of self-agency, but not the sense of self-ownership. Cognition, 94(3), 241-255.

Stouten, J., De Cremer, D., \& Van Dijk, E. (2005). All is well that ends well, at least for proselfs: Emotional reactions to equality violation as a function of social value orientation. European Journal of Social Psychology, 35(6), 767-783.

Stouten, J., De Cremer, D., \& Van Dijk, E. (2006). Violating equality in social dilemmas: Emotional and retributive reactions as a function of trust, attribution, and honesty. Personality and Social Psychology Bulletin, 32(7), 894-906.

Székely, M., \& Michael, J. (2018). Investing in commitment: Persistence in a joint action is enhanced by the perception of a partner's effort. Cognition, 174, 37-42. 
Tomasello, M. (2009). Why we cooperate: MIT press.

Tomasello, M., Melis, A. P., Tennie, C., Wyman, E., \& Herrmann, E. (2012). Two key steps in the evolution of human cooperation: The interdependence hypothesis. Current Anthropology, 53(6), 673-692.

Tversky, A., \& Kahneman, D. (1974). Judgment under uncertainty: Heuristics and biases. science, 185(4157), 1124-1131.

Wegner, D. M. (2004). Précis of the illusion of conscious will. Behavioral and Brain Sciences, $27(5), 649-659$. 


\author{
Appendix A \\ Sample of Written Guide on Training Trials Shown to Participants \\ TRAINING PHASE
}

For the training trials, you should aim to:

1. Get used to the hand device.

2. Reach the target within the first few seconds.

3. Keep your grip steady at target for another few seconds.

4. Experience your force at various target levels.

If you have any questions, feel free to ask before or at the end of the training phase. 


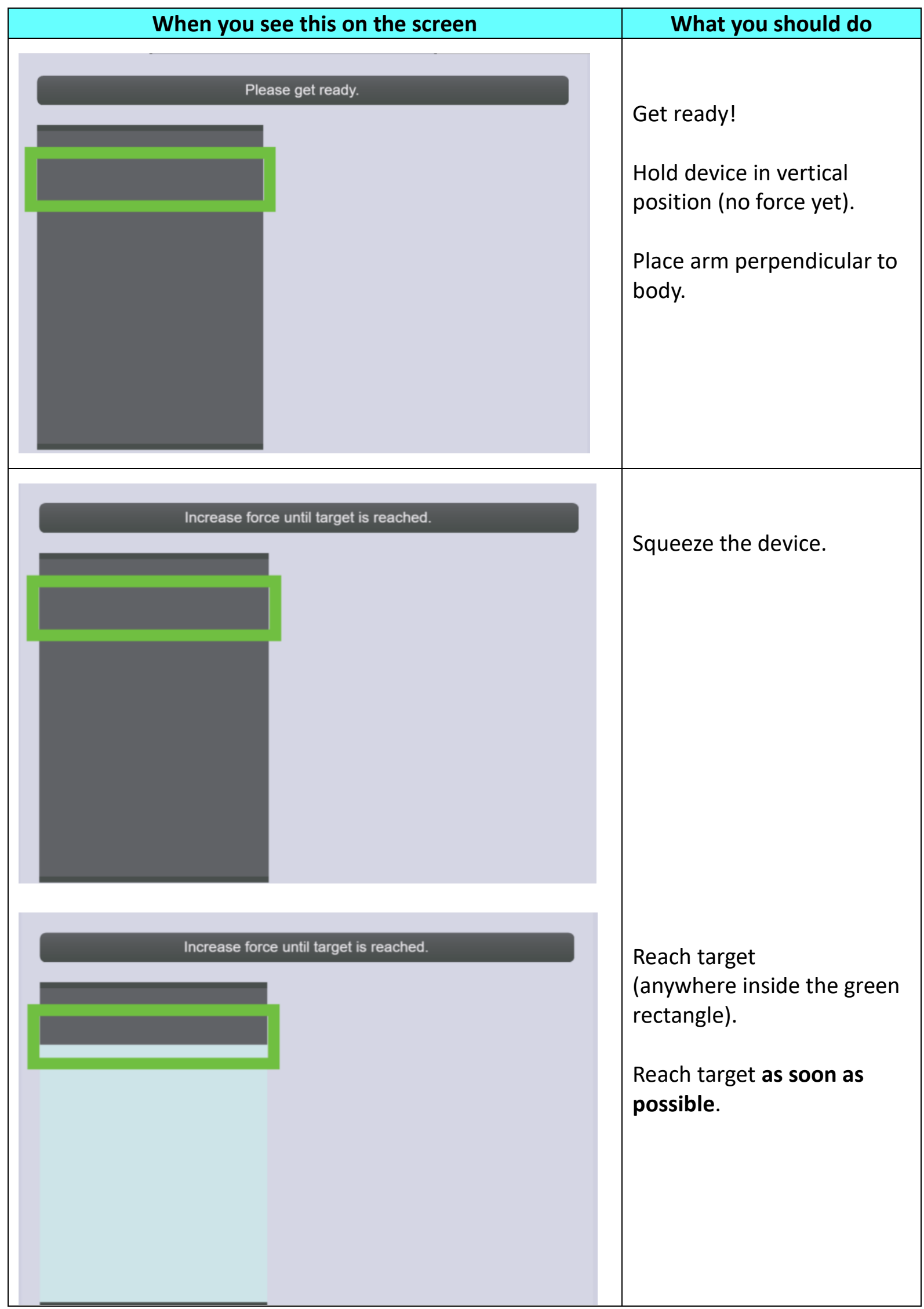




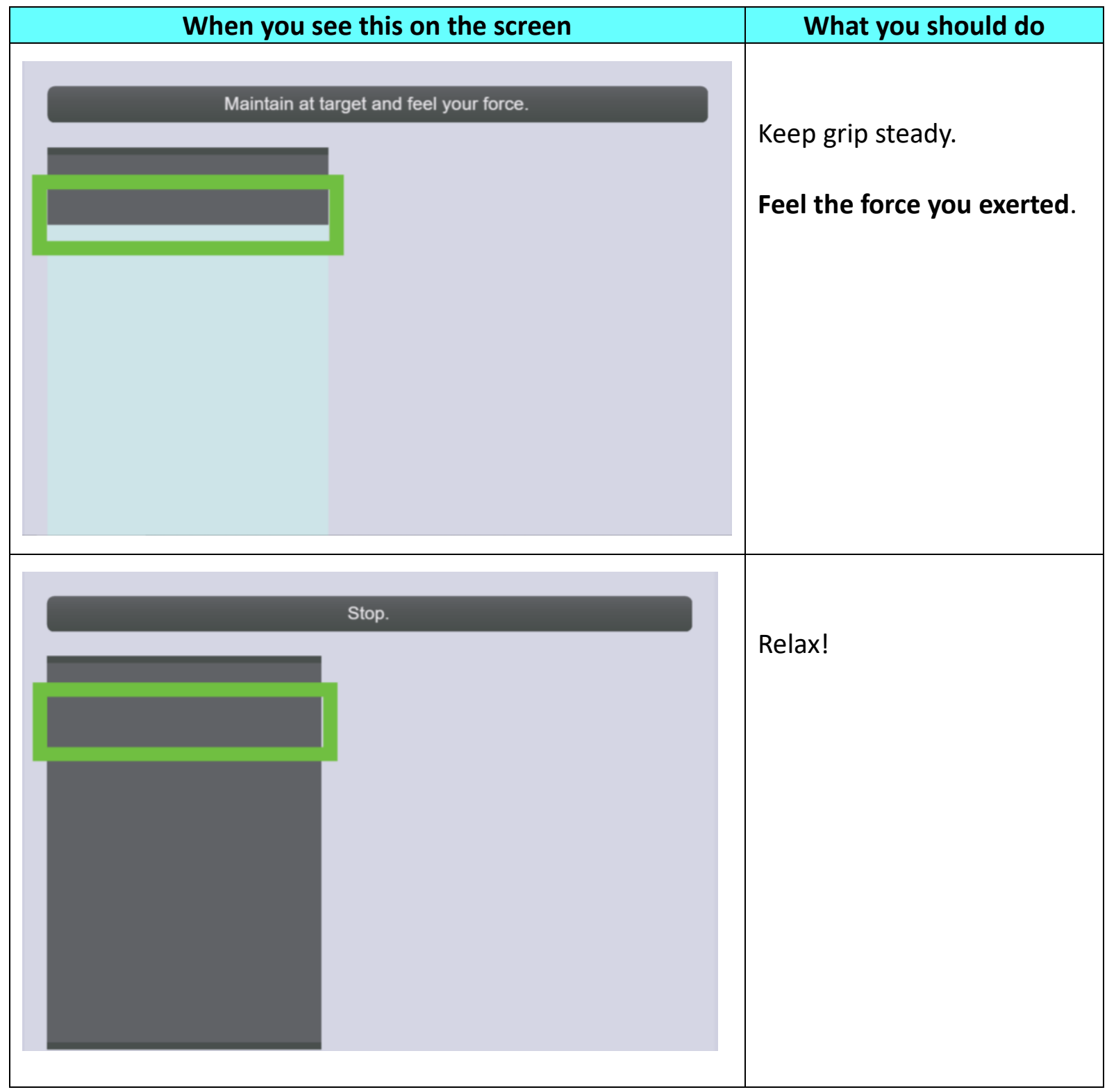


Appendix B

Sample of Written Guide on Experimental Trials Shown to Participants

\section{EXPERIMENT PHASE}

We will begin the experiment shortly.

You will be asked to squeeze the dynamometer in sets. In each set, there are 2 rounds.

\section{Round 1}

You will perform the task alone to reach the target. Feel the force you exerted in Round 1.

\section{Round 2}

You will repeat the task either:

(a) Alone, but with the COMPUTER adding some amount to the force you exerted;

or

(b) Together with your PARTNER seated across the table.

You will be doing both conditions (a) and (b) in this experiment. Please read the instructions on the screen carefully to know the condition you are in.

Feel the force you exerted in Round 2.

\section{Rating}

At the end of each set, you will be asked to evaluate your force exerted in Round 2, as a percentage of your force exerted in Round 1.

\section{Overall}

There are 12 sets in each block. You can take a break for as long as you need before proceeding with the next block.

We will run this experiment for a total of 4 blocks. 


\section{ROUND 1}

Similar to the tasks you just did in the training phase.

Remember to feel the force you exerted in Round 1. This is the baseline/reference point.

\section{ROUND 2}

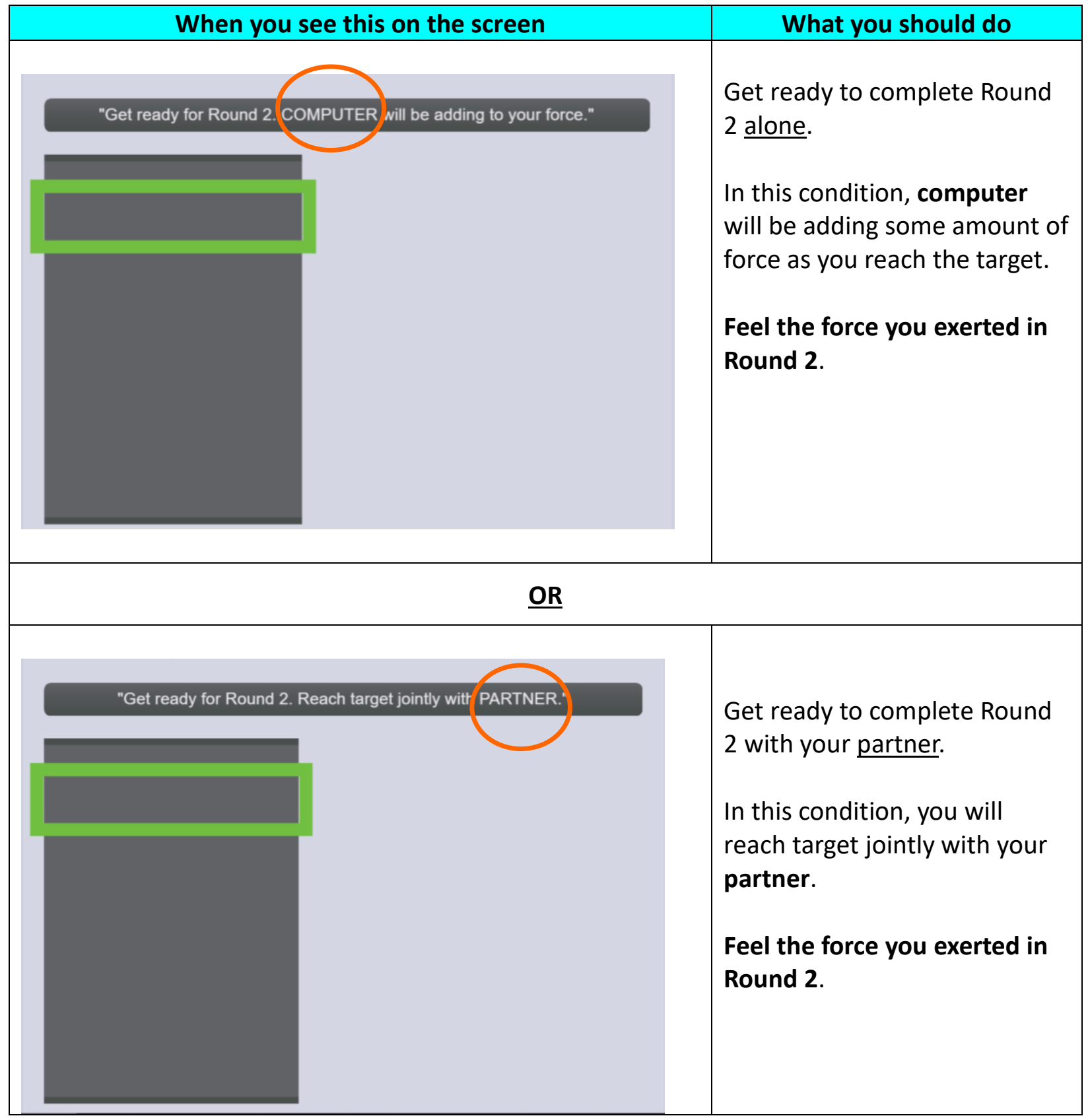




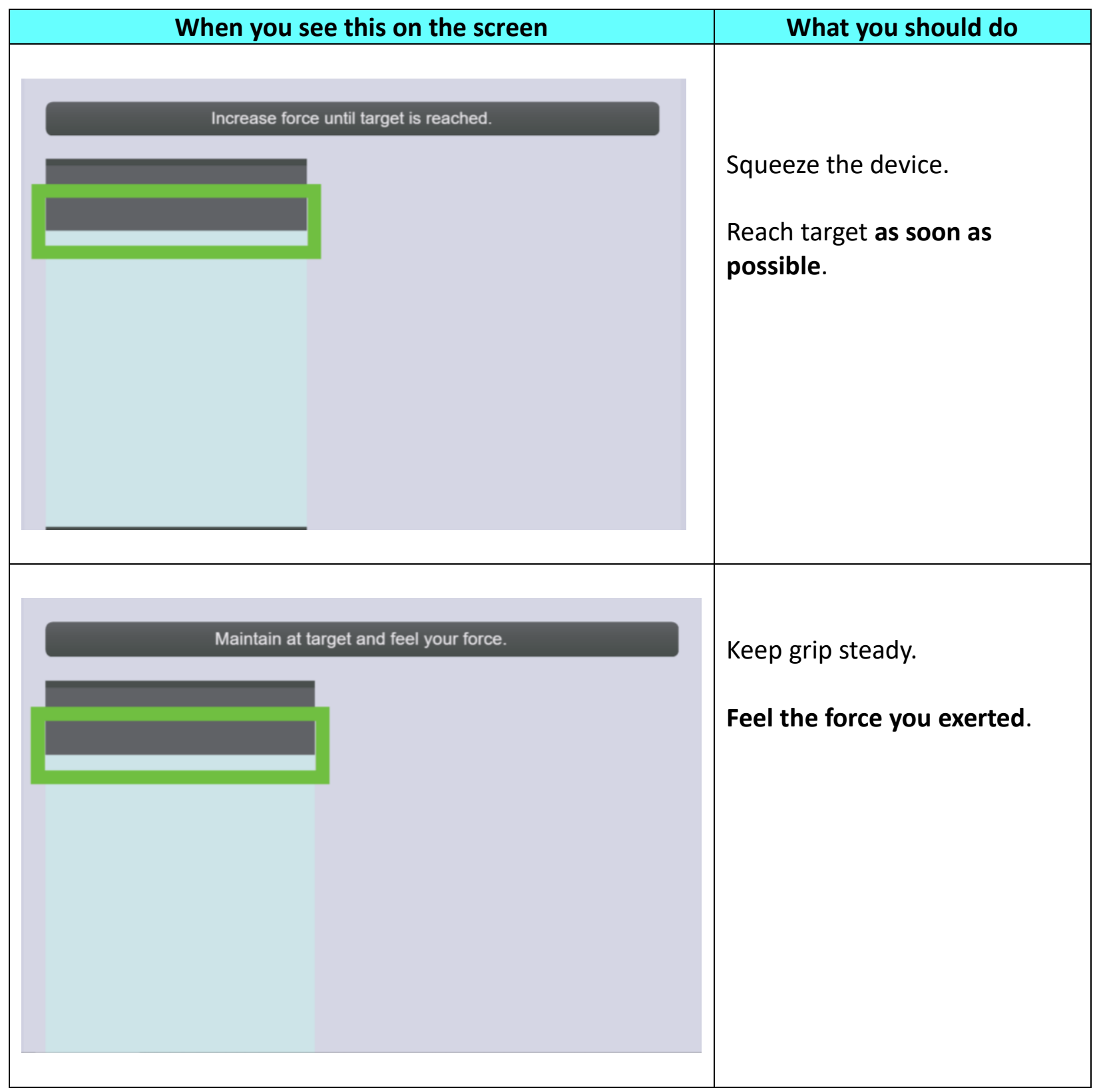




\section{RATING}

Rate $\frac{\text { Your force exerted in Round } 2}{\text { Your force exerted in Round } 1}$ expressed in percentage (\%)

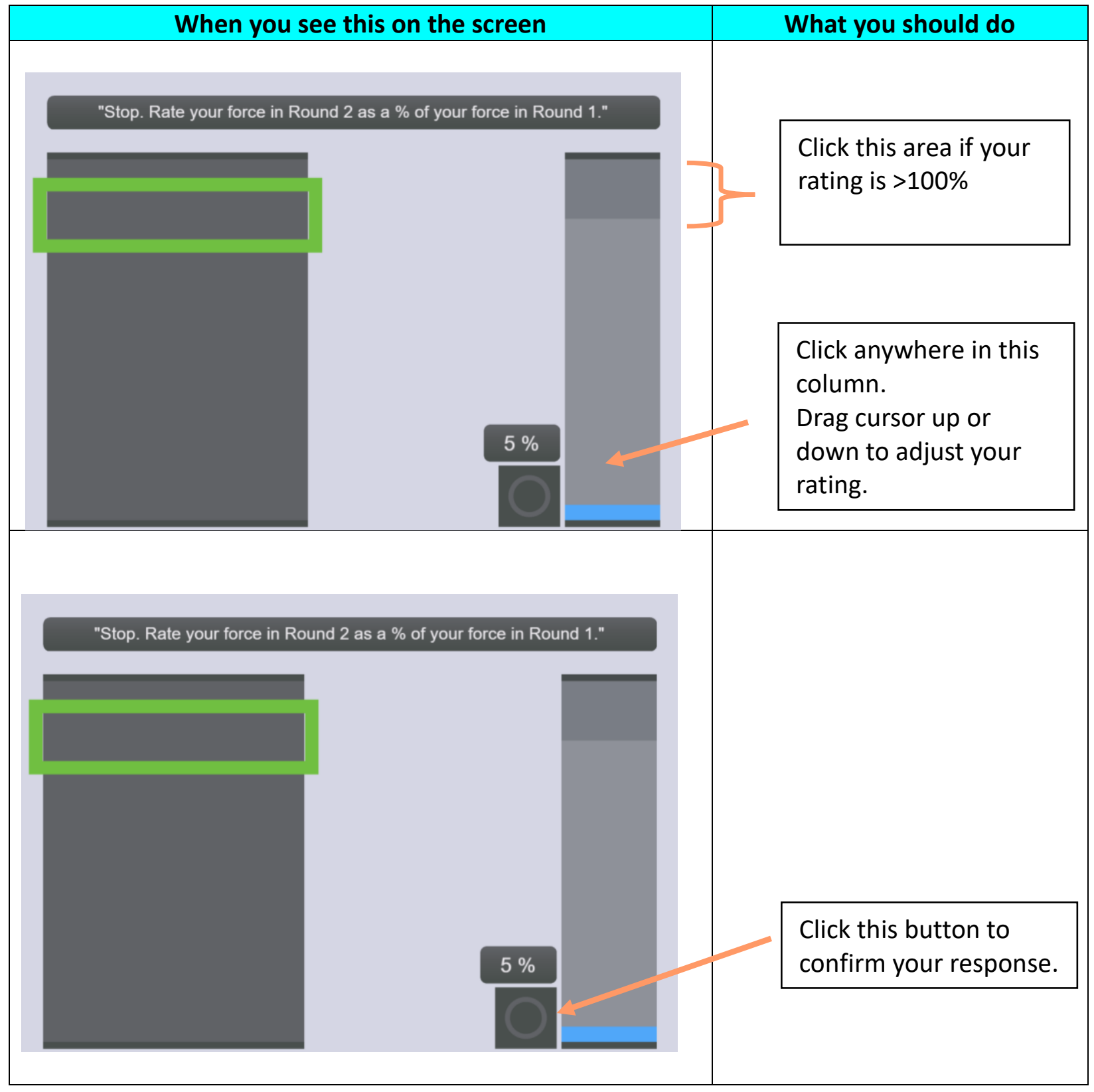




\section{PERFORMANCE}

Start exerting force once you see the message "Increase force until target is reached". Your force will be monitored from the time this message is shown until the time when you are prompted to stop.

The bonus will be based on your performance in reaching the targets as well as the accuracy in your ratings.

\section{END OF EACH BLOCK}

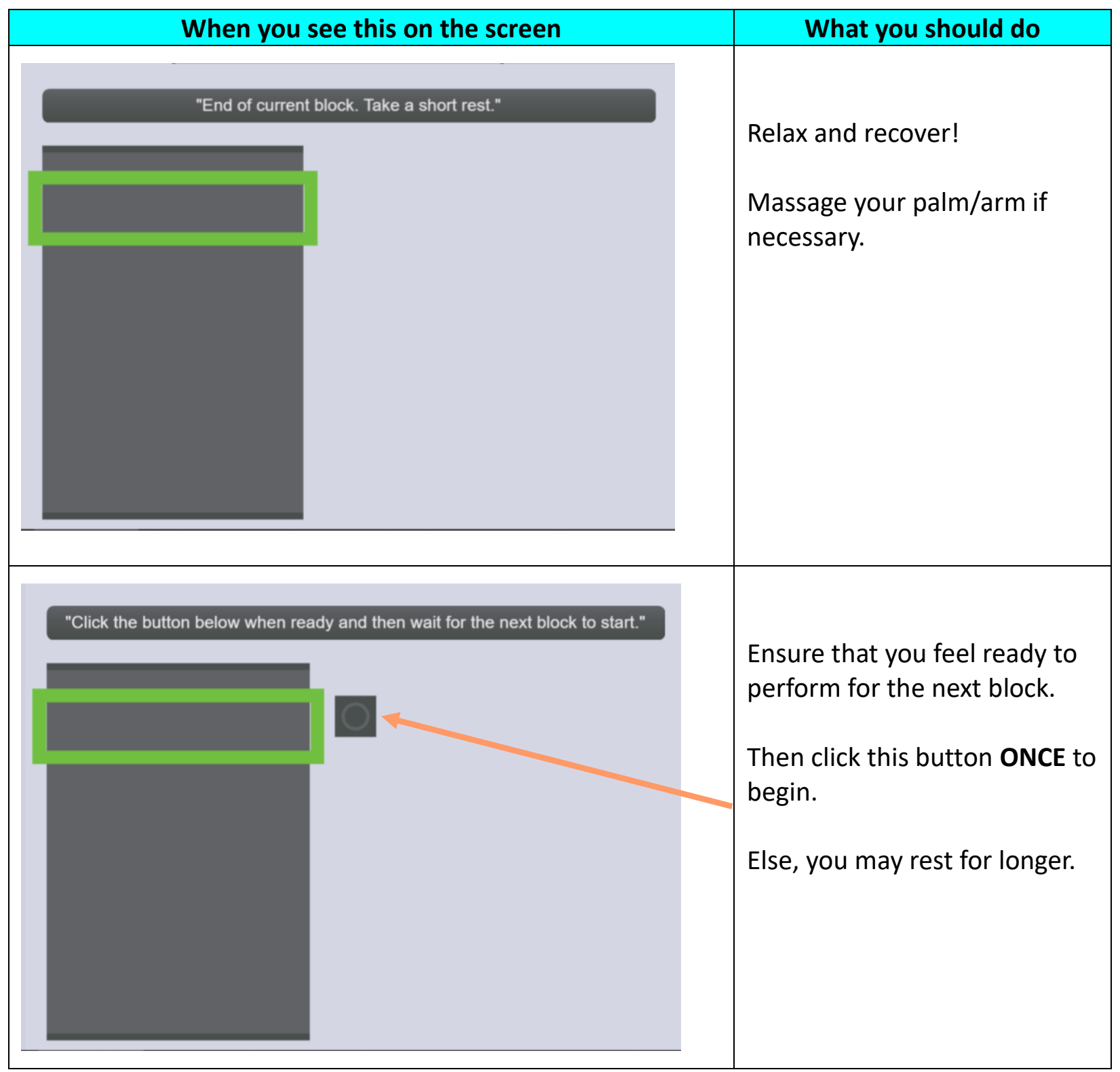

